\title{
Intersections
}

Canadian Journal of Music

Revue canadienne de musique

\section{De l’orchestre À la machine}

\section{François Gauthier}

Volume 33, numéro 1, fall 2012

URI : https://id.erudit.org/iderudit/1025557ar

DOI : https://doi.org/10.7202/1025557ar

Aller au sommaire du numéro

\section{Éditeur(s)}

Canadian University Music Society / Société de musique des universités canadiennes

ISSN

1911-0146 (imprimé)

1918-512X (numérique)

Découvrir la revue

Citer cet article

Gauthier, F. (2012). De l'orchestre À la machine. Intersections, 33(1), 83-101. https://doi.org/10.7202/1025557ar

\section{Résumé de l'article}

Le compositeur canadien Paul Baillargeon a réalisé la musique de plus de quarante épisodes des séries télévisées Star Trek, maintenant traduites et diffusées à travers le monde. Star Trek est l'une des dernières séries télévisuelles américaines ayant utilisé un orchestre symphonique d'envergure avant que ses principaux compositeurs réalisent eux-mêmes leurs bandes sonores à l'aide d'ordinateurs et d'échantillons numériques, remplaçant ainsi la cinquantaine de musiciens qui perpétuait la tradition acoustique de la production musicale à Hollywood. Cet article explore comment ces outils ont influencé la musique du compositeur, ainsi que son travail au sein de la postproduction des populaires séries télévisées.
Copyright @ C Canadian University Music Society / Société de musique des universités canadiennes, 2014
Ce document est protégé par la loi sur le droit d'auteur. L'utilisation des services d’Érudit (y compris la reproduction) est assujettie à sa politique d'utilisation que vous pouvez consulter en ligne.

https://apropos.erudit.org/fr/usagers/politique-dutilisation/ 


\title{
DE L'ORCHESTRE À LA MACHINE
}

\author{
François Gauthier
}

Le compositeur canadien Paul Baillargeon a réalisé la musique de plus de quarante épisodes des séries télévisées Star Trek, maintenant traduites et diffusées à travers le monde. Elles se situent parmi les dernières séries télévisuelles américaines ayant utilisé un orchestre symphonique avant que ses principaux compositeurs réalisent eux-mêmes leurs bandes sonores à l'aide d'ordinateurs et d'échantillons numériques. Nous explorons ici ces changements ainsi que la façon avec laquelle ils ont influencé la musique du compositeur ainsi que son travail au sein de la postproduction des populaires séries télévisées.

Star Trek consiste en un univers de science-fiction conçu et créé par Gene Roddenberry dans les années 1960. Il regroupe six séries télévisées, dont une série d'animations, onze longs métrages et des centaines de romans. Fred Steiner, l'un des concepteurs de la musique pour la série originale, a préféré l'usage d'une musique d'aventure au lieu d'une musique électronique futuriste et a mis l'accent sur l'aspect humain des personnages, sur l'héroïsme dont ils faisaient preuve en explorant la galaxie plutôt que sur le caractère technologique de la série ${ }^{1}$. L’orchestre utilisé pour un épisode d'une heure était généralement constitué d'un effectif de 25 à 30 musiciens ${ }^{2}$.

En ce qui concerne Star Trek: The Next Generation (1987), l'effectif fut porté à près de cinquante musiciens et a également été utilisé pour les séries suivantes: Deep Space Nine (1993); Voyager (1995) ; Enterprise (2001)3. En 2004, toutefois, les producteurs de Star Trek éliminèrent la formation orchestrale telle qu'elle existait depuis 1987 .

\section{L'OrChestre TraditionNel dANS LES SÉRIES STAR TreK}

Les budgets alloués à la musique pour la série The Next Generation (TNG) étaient considérables ${ }^{4}$ et les producteurs y accordaient une importance

1 Bond, 1998, p. 83.

2 Pour le premier épisode, The Cage, Alexander Courage compose (selon les différentes pièces) pour 3 flûtes, 1 hautbois, 2 clarinettes, 2 clarinettes basses, 3 cors d'harmonie, 3 trompettes, 3 trombones, 1 tuba, 1 guitare, 1 harpe, 1 orgue, 1 basse, 1 célesta, 4 percussionnistes et 1 soprano (voir Bond, p. 37).

3 Cela représente près de 700 épisodes comportant de la musique originale, toutes séries confondues.

4 Durant nos recherches en 2010, nous avons communiqué avec la Guilde des musiciens et musiciennes du Québec, puis avec le local 47 de l'American Federation of Musicians, basé à Los Angeles, pour connaître les coûts engendrés par ce type de production musicale, mais sans succès. Le superviseur de production que nous avons par ailleurs contacté, Peter Lauritson, n’a pas voulu dévoiler le montant des budgets alloués à la musique pour ces séries télévisées. C’est le compositeur et chef 
particulière, notamment à cause de l'influence que la musique des longs métrages de Star Trek avait produite à cette époque.

Quand nous avons commencé [la série The Next Generation], ils avaient déjà produit trois longs métrages 5 avec les personnages de la série originale des années 6o. Les films étaient grandioses et quand on a commencé à produire TNG, les producteurs Gene Roddenberry, moi-même et Bob Justman, avons décidé que ce serait formidable si nous pouvions avoir le son d'un orchestre complet pour donner ce sentiment de puissance et de majesté à une émission de télévision qui se déroule dans l'espace ${ }^{6}$. (Richard Berman, communication personnelle, 24 novembre 2010)

Toutefois, pour les séries télévisées, le producteur exécutif Rick Berman préférait utiliser la musique avec parcimonie et discrétion. Le succès rencontré par l'émission pilote Encounter at Farpoint (1987) fixa en quelque sorte l'effectif de l'orchestre, un mécanisme fréquent en télévision.

Pour les séries télévisées, la dimension de l'orchestre et le temps alloué pour enregistrer un épisode sont établis une fois passée l'étape de l'émission pilote. Lorsqu'un orchestre est utilisé et payé par la compagnie de production, on annonce au compositeur quel sera l'effectif de l'orchestre et le nombre d'heures prévues [financièrement] pour l'enregistrement. En 2002, par contre, il y avait très peu de séries télévisées comportant un orchestre de quelques dimensions que ce soit 7 . La musique de la plupart des émissions était réalisée par le compositeur lui-même avec peut-être un musicien ou deux ${ }^{8}$. (Karlin \& Wright, 2004) ${ }^{9}$

Ce commentaire reflète ce qui s'est produit chez Paramount concernant Star Trek. Bien que l'ampleur de l'orchestre utilisé pour la série télévisée fut moins important que pour les longs métrages, l'effectif instrumental était tout de même constitué d'une cinquantaine de musiciens par épisode et pour chaque épisode, les compositeurs des différentes séries réalisèrent des bandes musicales originales.

d'orchestre Simon Leclerc qui a affirmé, lors d'un entretien, que ces montants étaient importants par rapport aux productions courantes.

5 Il s'agit de Star Trek: The Motion Picture (1979), The Wrath of Khan (1982) et The Search for Spock (1984). La production télévisuelle de The Next Generation fut annoncée officiellement le 10 octobre 1987, 17 ans après la fin de la série originale, tandis que le $4^{\mathrm{e}}$ long métrage The Voyage Home est sorti en salle aux États-Unis le 26 novembre 1986. (Site Internet Forgotten Trek, http://www.ottens.co.uk/ forgottentrek/tng_8.php, consulté le 31 janvier 2012).

6 "When we started, they had already produced three feature films with the original Star Trek people from the 6o's. The movies were very grand and when we started doing TNG, producers Gene Roddenberry, myself and Bob Justman, all decided that it would be great if we could have a full orchestral sound to give that sense of power and majesty to a show that takes place in outer space.» Toutes les traductions sont de l'auteur.

7 Ce sont Karlin et Wright qui soulignent.

8 "In episodic television, the orchestra size and time allotted to record a show are pretty much locked in once the series gets past the pilot stage. When there will be an orchestra of some sort being paid by the production company, the composer is told what the orchestra makeup is and the number of hours budgeted for recording. In 2002, though, there were very few shows with orchestra of any size. Most shows will be self-performed by the composer with perhaps an occasional live musician or two.»

9 Karlin \& Wright 2004, p. 51. 
Le noyau de l'orchestre était stable et formé généralement par six cors français et une trentaine de cordes. Les autres cuivres et les vents, ainsi que différents instruments comme la harpe, les percussions ou un synthétiseur, complétaient la formation selon les besoins des compositeurs. Ce sera ce type d'orchestre que Paul Baillargeon devra éventuellement simuler à l'aide d'ordinateurs et d'échantillons numériques.

Est-ce qu'il existait un "son Star Trek» pour ces séries? La plupart des personnes interviewées pour la recherche, ainsi que d'autres commentateurs, répondent oui. Pour s'en assurer, il faudrait analyser une sélection représentative d'un corpus de près de 300 heures de musique pour se faire une idée précise de l'ensemble des musiques composées et produire un véritable travail à ce sujet, ce qui reste à accomplir ${ }^{10}$. Pour Jeff Bond, les musiques produites au sein des épisodes de l'ensemble des séries se sont transformées au fil des ans ${ }^{11}$.

Star Trek: The Next Generation s'est développé en un tout cohérent, et sa musique s'est, pour la première fois, liée organiquement avec les images, le jeu des acteurs et le ton dramatique de la série. Finalement [Jay] Chattaway s'est adapté aux goûts des producteurs, et la musique des trois dernières saisons de cette série a atteint une cohérence stylistique qui n'existait pas à l'époque de [Dennis] McCarthy et de [Ron] Jones ${ }^{12}$. (Bond, 1998)

Il est toutefois intéressant de noter que pour Richard Berman, il n'y avait pas vraiment de son Star Trek, ni même une sonorité caractéristique qu'un public, même averti, aurait pu reconnaître, à l'exception des indicatifs musicaux de chaque épisode. Il explique ainsi l'homogénéité apparente de la sonorité de la musique des séries:

Il y avait l'ingénieur, il y avait Peter Lauritson qui était toujours là [aux séances d'enregistrement et du mixage audio], il y avait toujours quelqu'un comme Paul [Baillargeon] ou Dennis [McCarthy] pour diriger l'orchestre, puis les personnes responsables du mixage définitif étaient toujours les mêmes. Le son avait la même qualité. La seule chose qui variait c'étaient les éléments créatifs de la composition, qui variaient d'un compositeur à l'autre. ${ }^{13}$ (Richard Berman, communication personnelle, 24 novembre 2010)

10 Nous calculons cette approximation en considérant qu'il existe à peu près 700 épisodes de Star Trek, toutes séries confondues, comportant une moyenne de 25 minutes de musique par épisode.

11 Bond 1998, p. 171.

12 «Star Trek: The Next Generation had mature into a cohesive whole, and its musical scores for the first time combined organically with the images, performances and dramatic tone of the series. Eventually Chattaway adapted to the tastes of the producers, and the scores of The Next Generation's final three seasons achieved a stylistic coherence that had always evaded the days of McCarthy and Jone's tenure.»

13 "You had the engineer, you had Peter Lauritson who was always there, you always had someone like Paul or Dennis conducting, and then the guys who did the final mixes were always the same. The sound had the same quality to it. The only thing that varied was the creative elements of composition, which varied from composer to composer.» 


\section{DE L'ANALOGIQUE AU NUMÉRIQUE}

Les séries Star Trek, de l'originale en 1967 à Voyager qui se termine au mois de mai 2001, ont été populaires et lucratives. Le vent commence à tourner avec la dernière série de la franchise, Enterprise (2001). Dans une entrevue avec Jennifer Howard, réalisée pour le compte d'Archive of American Television ${ }^{14}$, le producteur exécutif et l'auteur de la série, Richard Berman, raconte que les cotes d'écoute commencent à diminuer significativement au courant de la deuxième saison. Il attribue ce déclin à ce que la direction de Paramount appelle franchise fatigue.

Malgré les efforts de Berman et de son collègue Brannon Braga pour rétablir la situation, l'intérêt des téléspectateurs de la série diminuait, et conséquemment les revenus publicitaires aussi. Ces producteurs ont alors convenu de réduire considérablement l'effectif de l'orchestre. Mais, bien qu'il s'agisse principalement d'une question financière, on ne peut négliger le rôle qu'a joué la qualité des échantillons numériques dans cette décision. Nous avons demandé à Richard Berman ce qu'il en pensait. L'exemple que nous donna Berman concerne les technologies visuelles, mais s’applique également à la musique.

Lorsque nous avons commencé à produire Star Trek: The Next Generation (1987), nous avons construit des modèles qui ont été filmés dans un studio d'effets spéciaux pour les objets qui étaient dans l'espace. Nous avons utilisé des modèles au lieu du numérique parce qu’obtenir la même qualité à l'aide de l'animation assistée par ordinateur était beaucoup trop dispendieux. Il n'y avait aucune façon de le faire afin d'obtenir d'aussi bons résultats. Et soudainement, comme les années passaient, en quatre ou cinq ans, le prix pour utiliser les systèmes de génération d'images par ordinateur était plus abordable. Nous avons constaté que nous pouvions produire des images numériques d'aussi bonne qualité que les modèles, pour le même montant d'argent, puis éventuellement pour moins d'argent que les modèles. Alors, nous avons complètement cessé d'utiliser des modèles et tout a commencé à être généré uniquement par des ordinateurs. C'était aussi vrai en ce qui concerne la musique. La qualité des échantillons et les différentes techniques de synthèse dont vous parlez étaient soit trop dispendieuses ou pas d'assez bonne qualité. Et comme les années passèrent, la technologie s'est améliorée et puis tout d'un coup nous pouvions obtenir des produits de très bonne qualité pour moins d'argent, ce que le studio voulait que nous fassions, dépenser moins d'argent ${ }^{15}$. (Richard Berman, communication personnelle, 24 novembre 2010)

14 Rick Berman, Archive of American Television, <http://www.emmytvlegends.org/interviews/ people/rick-berman>, consulté le 28 mars 2013.

15 «When we first started to make Star Trek: The Next Generation (1987), for the stuff that was out in space, we built models that were photographed in a special effects studio. The reason we used models as oppose to digital, was because to get the same quality using digital generated animations was way too expensive. There was no way we could do it and have it look as good. And all of a sudden, as the years went by, four or five years, the price of doing digital visual work, CGI, got cheaper. We found that we could do computer generated imaging that looked as good as the models have looked, for the same amount of money, and eventually for less money than models. So models stopped being used entirely and everything started being generated by computers. The same thing was true with music. The quality of the samplings and the various synthetic techniques that you are talking about were 
Ces deux éléments, la qualité des échantillons numériques et les coûts de production étaient intimement liés. À la fin de la troisième année de la série Enterprise, les producteurs de l'émission ont annoncé aux compositeurs qu'ils réduisaient l'orchestre à une quinzaine de musiciens. «Quinze musiciens! On part d'une cinquantaine. Je n'ai aucune idée comment m'y prendre pour que ça sonne de la même façon", s'exclama Baillargeon, qui pourtant n'en était pas à sa première rencontre avec les ordinateurs et les synthétiseurs ${ }^{16}$. Mais, faire sonner un orchestre synthétique de façon presque identique à celui utilisé pour Star Trek, voilà un nouveau défi qu'il relèvera avec succès.

Il s'est procuré l'équipement nécessaire à l'été 2004, où il a exploré les différentes possibilités offertes par les diverses composantes, c'est-à-dire un ordinateur Apple, le séquenceur multipiste Digital Performer et des banques d'échantillons haut de gamme comme celles d'EastWest et de Vienna. "J'ai passé l'été à pratiquer avec mes machines", dit-i ${ }^{17}$. Il avait sélectionné un top réalisé à l'orchestre l'année précédente et le reproduisit à l'aide de son nouvel équipement ${ }^{18}$. Lorsqu'il termina le top numérique, il le fit parvenir au superviseur de production, Peter Lauritson, qui trouva le résultat excellent. Baillargeon décida alors de ne travailler qu'avec les machines, contrairement à ses collègues Dennis McCarthy et Jay Chattaway ${ }^{19}$.

Toutefois, préparer et produire le test de simulation pour Peter Lauritson a donné du fil à retordre à Baillargeon. Avant de reproduire la partition à l'aide des machines, il avait invité à son domicile Richard Winquest, son propre ingénieur du son pour les séries Star Trek, afin de préparer l'équipement et disposer les instruments de l'orchestre virtuel de façon à simuler avec exactitude l'emplacement des vrais musiciens. Le système utilisé permettait à Winquest de placer les «microphones» aux endroits désirés dans le champ stéréophonique. Ce fut avec cet orchestre virtuel que Baillargeon composa la musique de trois épisodes d'Enterprise durant la dernière année de la série, en $2005^{20}$. Comme il l'explique aujourd'hui, cela n'a peut-être rien changé «dans la vraie vie». Qui s'est réellement rendu compte que la clarinette ou les cors se situaient

either too expensive or not quite good enough. And as the years went by, the technology got better and better and all of a sudden we were getting really good quality stuff for less money, which is the studio wanted us to do, spend less money.»

16 Paul Baillargeon, communication personnelle, 17 septembre 2009.

17 Par «machines», nous entendons un ensemble d'appareils, quelle que soit leur combinaison, comme des échantillonneurs, des synthétiseurs, physiques ou virtuels et des ordinateurs qui servent à utiliser différents logiciels de productions musicales ou ceux qui gèrent les diverses banques de sons numérisés offerts sur le marché. Cela inclut tous les appareils périphériques qui permettent de manipuler, de traiter et de sauvegarder les sons ou les données nécessaires à leur utilisation, ainsi que les techniques requises pour permettre la communication entre ces divers appareils. Ce terme, que l'on peut utiliser dans son sens général de système où existe une correspondance spécifique entre une information d'entrée et celle de sortie, ou encore comme dispositif de saisie, de traitement ou d'exploitation de l'information (Le Petit Robert 2011, Paris, France: Dictionnaire Le Robert, p. 1497), a souvent été utilisé par les personnes consultées pour la réalisation de cet article. Nous ferons de même ici.

18 Malgré de nombreux efforts de la part du compositeur, il n'a pas été possible pour lui de retrouver l'enregistrement de ce top, réalisé à l'aide d'échantillons numériques, afin qui nous puissions le comparer à celui de l'orchestre.

19 Paul Baillargeon, communication personnelle, 27 août 2009.

20 Deadalus (4.086), Babel One (4.088) et Demons (4.096). 
à la bonne place ou non? Paul Baillargeon avait toutefois l'intention d'être le plus fidèle possible à l'espace sonore de l'orchestre réel qu'il connaissait, et cela l'a aidé à poursuivre son travail ${ }^{21}$.

Par ailleurs, ce travail de simulation n'avait rien d'aisé. Baillargeon avait consacré quatre à cinq heures pour composer le top original et cela avait pris une quinzaine de minutes pour l'enregistrer avec l'orchestre lors de la séance d'enregistrement aux studios Paramount de Los Angeles. Le compositeur a toutefois dû consacrer une cinquantaine d'heures pour réaliser le même top à l'aide des machines ${ }^{22}$. La même chose se produisit concernant les premiers pas de Dennis McCarthy avec les appareils numériques. Moins à l'aise avec ces derniers, il a mandaté un programmeur pour réaliser un top qu'il avait composé pour l'occasion. Le programmeur a pris une centaine d'heures pour accomplir sa tâche de façon satisfaisante ${ }^{23}$.

Ces anecdotes documentent de façon intéressante l'impact des technologies numériques sur le travail du compositeur. Malgré le degré élevé de perfectionnement des appareils utilisés, c'est-à-dire un ordinateur performant, un logiciel de production musicale à la fine pointe des technologies audio, la qualité élevée des échantillons numérisés et un éventail important d'articulations, il devenait extrêmement complexe pour Baillargeon et ses collègues de reproduire avec fidélité une musique riche et subtile comme celle qu'ils composaient pour un véritable orchestre. Bien qu'il trouvait que Paul Baillargeon travaillait habilement, Peter Lauritson se rappelle ainsi cette transition difficile vers le numérique et les premiers résultats.

Ce ne fut pas ce qu'il y avait de plus satisfaisant, c'était un genre de mal nécessaire. C'était un nouveau territoire pour tous nos compositeurs et ils essayaient très fort, mais c'était juste correct. Ça s'est définitivement amélioré avec le temps, mais il y a eu quelques déceptions concernant les premières compositions numériques ${ }^{24}$. (Peter Lauritson, communication personnelle, 5 décembre 2010)

Le premier épisode de la série Enterprise pour lequel Paul Baillargeon écrivit une musique $100 \%$ électronique s'intitulait Daedalus et fut diffusé le 14 janvier 2005. Baillargeon avait choisi de travailler seul, sans la douzaine de musiciens mis à sa disposition, contrairement à ce que ses collègues McCarthy et Chattaway ont fait, parce qu'il estimait le degré de difficulté trop important pour simuler l'orchestre à l'ordinateur en plus de le jumeler à de vrais instrumentistes. La qualité des échantillons avec lesquels il a expérimenté pendant quelques mois rendait, selon lui, l'effort superflu. Il ne voyait pas pourquoi ajouter, par exemple, un seul cor d'harmonie réel à un ensemble de six cors virtuels. C'était

21 Paul Baillargeon, communication personnelle, 17 septembre 2009.

22 Pierre-Daniel Rheault, communication personnelle, 11 août 2009.

23 Simon Leclerc, communication personnelle, 19 novembre 2009. Simon Leclerc est compositeur et chef d'orchestre. Il a dirigé l'orchestre de Paramount à plusieurs reprises lors des enregistrements de la musique de Paul Baillargeon pour Star Trek.

24 «It was not all that satisfying, it was kind of a necessary evil. It was new territory for all our composers and they were trying very hard but it was just OK. It definitely got better as we progressed, but in the first few [digital scores], there were some disappointment.» 
beaucoup plus facile pour lui de réaliser en entier un top à l'aide de ses appareils, que de séparer la tâche en deux étapes. Il reconnaît toutefois être certain qu'avec de vrais musiciens, il aurait écrit une musique qu'il ne ferait pas avec les échantillonneurs, parce qu'avec les machines, dit-il en souriant, «cela prendrait une journée pour faire deux mesures ${ }^{25}$ ».

Il n'était donc pas économiquement rentable d'investir beaucoup de temps dans la création d'une musique sophistiquée, qui, elle, était beaucoup plus facile à produire avec un orchestre réel. Le passage de l'analogique au numérique s'est échelonné sur plusieurs mois et la compagnie Paramount a dû réorganiser ses processus de postproduction. Certains ne changèrent guère, comme les séances de pointage, mais d'autres se transformèrent sensiblement tandis que l'un des plus importants, la séance d'enregistrement de l'orchestre, disparut tout simplement.

\section{UN ORCHESTRE AU BOUT DES DOIGTS}

En 1988, l'ingénieur du son Doug Rogers fonde la compagnie EastWest afin de produire des échantillons numériques pour les musiciens. Au milieu des années 1990, Rogers se joint au compositeur Nick Phoenix et met sur pied Quantum Leap, une division d'EastWest qui deviendra l'un des plus importants producteurs de banques de sons et d'instruments virtuels dans le monde.

Le système PLAY ${ }^{26}$ fourni avec les banques d'échantillons est un logiciel qui permet d'ouvrir et de jouer des fichiers de sons numérisés provenant de différentes banques. Il est constitué d'une interface graphique qui permet l'accès à différents instruments et à chaque instrument est associée une quantité variable d'échantillons. Un des éléments essentiels de ce logiciel consiste à permettre la reproduction des principales articulations d'un instrument de musique. La reproduction fidèle des modes de jeu est essentielle dans la simulation de l'orchestre et la qualité du rendu s'est grandement améliorée depuis les années 1990. On retrouve donc, pour chaque instrument, différents modes de jeu comme le jeu soutenu, le staccato, le trille du violon, le pizzicato Bartók, etc. Les nuances dynamiques d'un instrument font partie des modes de jeu. Selon la banque de sons utilisée, différents niveaux dynamiques sont attribués à chaque échantillon de chaque instrument.

Mais il faut plus que de simples échantillons de sons d'orchestre pour parvenir à produire un jeu instrumental qui s'approche de celui d'un tel ensemble. Un bon son, évidemment souhaitable, ne suffit pas. Il faut donner l'impression du jeu instrumental réel pour faire sonner pareil l'orchestre de la machine et l'orchestre réel, car, le jeu instrumental, c'est le style de l'instrumentiste ${ }^{27}$. En cela, les technologies d'échantillonnage sont de précieux outils. Ils permettent de compartimenter virtuellement presque tous les éléments mis en œuvre par un musicien jouant de son instrument, et de les reconstituer selon les besoins

25 Paul Baillargeon, communication personnelle, 17 septembre 2009.

26 Nommé PLAY Advanced Sample Engine dans le manuel d'instruction du fabricant (20072010).

27 Heinrich 2003, p. 56. 
du compositeur. En isolant et en standardisant ces éléments (dynamique, volume, geste, position virtuelle des microphones, fondu sonore d'une note à l'autre, etc. $)^{28}$, des logiciels et des banques de sons comme EastWest permettent aujourd'hui à divers musiciens de produire une musique qui sonne bien, s'ils y consacrent le temps nécessaire. Paul Baillargeon s'exprimait ainsi à ce sujet:

Je pense qu'avec les machines d'aujourd'hui, nous pouvons [musicalement] faire des choses relativement convenables. Vous devez être très patient et vous devez savoir écrire. Mon avantage, c'est que j'ai composé beaucoup de pièces pour orchestre, et quand je travaille avec des machines, au moins j'ai une idée de ce que j'essaie de faire. Et régulièrement je retourne écouter d'authentiques musiques [d'orchestre], car vous pouvez vous laisser aisément berner [par les machines] ${ }^{29}$. (Paul Baillargeon, conférence du 2 novembre 2008, Université de Montréal)

Baillargeon a souligné l'importance d'être patient avec ces outils informatiques puisqu'il savait très bien que le travail de composition et la mise au point du résultat sont longs à effectuer, du moins pour le style de musique qu'il devait créer pour Star Trek. Les technologies utilisées ici rendaient le travail de création plus complexe. Par ailleurs, comme le suggère Baillargeon, il est préférable de savoir déjà écrire pour un orchestre, c'est-à-dire de connaitre les possibilités de chaque instrument, ses forces et ses faiblesses, les sonorités qu'ils produisent lorsqu'ils sont joués ensemble, etc., parce qu'il est facile de s'éloigner du son authentique de l'orchestre si l'on ne se réfère qu'à ce que les échantillons numériques nous offrent.

\section{SUBTILES MUTATIONS}

Pour Émile Leipp, cité par Marie-Noëlle Heinrich ${ }^{30}$, «l’instrument de musique est une machine à fabriquer des sons ». Il faut bien reconnaître, comme le mentionne l'auteure, que les instruments de musique mis au point au fil du temps proviennent des efforts que l'humain a accomplis pour maîtriser des problèmes considérables d'ordre acoustique et technologique. Ces instruments traditionnels transforment l'énergie mécanique en énergie vibratoire aérienne, et relèvent ainsi des problèmes liés au domaine de la mécanique des solides et des fluides. La relation physiologique entre l'instrumentiste et sa machine, caractérisée par une gestuelle propre à chaque instrument de musique et à chaque interprète, est importante. Cette relation change avec les instruments virtuels de studio. Les machines qui permettent de jouer de ces instruments servent surtout, en fait, à manipuler des paramètres acoustiques ${ }^{31}$.

28 La réalisation de banques de son de qualité requiert une expertise technique importante.

29 «I think that with the machines today, we can do relatively nice stuff. You have to be really patient and you have to know how to write. My advantage is that I did a lot of orchestral work so, and when I am working with machines, at least I have an idea of what I am trying to do. And regularly I will go back and listen to real cues because you can get fooled so badly."

30 Heinrich 2003, p. 46.

31 Ibid., p. 55. 
Auparavant, Paul Baillargeon écrivait sur du papier à musique en spécifiant tous les modes de jeu que les musiciens interprétaient lors de la séance d'enregistrement. Maintenant, avec ce système d'échantillons, le compositeur doit non seulement choisir et valider l'articulation appropriée, c'est-à-dire qu'il doit vérifier quelles articulations sont disponibles sur telle ou telle banque, en plus de déterminer si elles lui conviennent ou non, mais il doit aussi la jouer lui-même ou indiquer au séquenceur comment le faire. Le compositeur assume désormais la tâche de l'instrumentiste, car c'est son jeu à lui, ou sa propre programmation, qui se retrouve dans la version définitive d'un top. Bien que Baillargeon compose pour les divers instruments de l'orchestre depuis de nombreuses années et qu'il connaît leurs caractéristiques, les reproduire par programmation ou les simuler de façon réaliste à l'aide d'échantillons était exigeant. Ce commentaire de Richard Winquest souligne les difficultés du jeu instrumental en situation réelle.

Ce n'est pas facile de faire un bon solo de violon, parce que vous êtes limité, car vous ne pouvez faire que certaines choses au synthétiseur, car ça ne sonne tout simplement pas comme il faut. Comme tu le sais, elles [les machines] s'améliorent et certaines personnes sont meilleures que d'autres [pour en jouer]. Mais un grand nombre d'instruments sont vraiment difficiles à jouer en $\operatorname{solo}^{32}$. (Richard Winquest, communication personnelle, 15 janvier 2010)

Une fois un top créé, il sera joué par les machines de façon identique, à tout coup. La machine interprète les mêmes paramètres à partir des mêmes sources sous les mêmes conditions. Le contrôle est parfait. Mais ces paramètres, bien que nombreux, sont complètement standardisés. D’une certaine façon, le compositeur n'est plus en relation avec les instruments eux-mêmes, comme lorsqu'il dirige ou qu'il écoute l'orchestre interpréter sa propre musique. Il s'est éloigné des véritables instruments, mais aussi de ceux qui les maniaient. Comme le souligne Heinrich, c'est à travers des dispositifs intermédiaires qu'il prend conscience des résultats de son travail: clavier, souris, écran, interface graphique, haut-parleurs. Ultimement, la relation du geste à l'instrument disparaît ${ }^{33}$. En fait, elle se transforme, puisqu'elle est maintenant uniformisée. Par exemple, un coup d'archet joué sur une même note avec la même articulation et la même vélocité utilisera toujours le même échantillon de violon d'une banque de sons déterminée.

À première vue, cela n'a rien changé au processus de composition lui-même, puisque Baillargeon créait auparavant sa musique en connaissant bien les capacités de l'orchestre. Avec les machines, il n'avait qu'à sélectionner et appliquer les différents paramètres offerts par le logiciel pour reproduire virtuellement l'orchestre. Il jouait lui-même la musique de chaque instrument à l'aide de son

32 «It's not easy to make a really nice violin solo, because you are limited, because you can only do certain things on synth, because it just doesn't sound right. As you know, it's [les machines] getting better and some people are better at it than other people. But a lot of instruments are really tough on the solo position.»

33 Heinrich 2003, p. 203. 
clavier, comme il le faisait lorsqu'il écrivait sur du papier à musique ${ }^{34}$. Mais la réalité est plus complexe, car écrire à l'aide d'un ordinateur et d'échantillons numériques peut entraîner de subtils changements de comportement, comme le fait remarquer le compositeur et chef d'orchestre Simon Leclerc ${ }^{35}$.

Si tu sais que le produit définitif sera entièrement fait aux machines, tu vas t'organiser pour utiliser ce qui sonne le mieux avec les sons que tu as. Ce qui n’a pas nécessairement rapport avec un instrument acoustique. $\mathrm{Si}$ tu sais que ton basson sonne très bien dans le registre extrême grave et dans le registre extrême aigu, mais qu'il ne sonne pas bien dans le registre moyen, tu ne le feras jamais jouer dans le registre moyen, alors que le vrai basson est absolument magnifique dans ce registre. (Simon Leclerc, communication personnelle, 19 novembre 2009)

Si différents types de phrasés, d'articulations ou de modes de jeu particuliers des machines ne répondent pas bien, le compositeur se limite, utilisera d'autres expressions musicales, prendra d'autres décisions. Il est possible que ce subtil changement d'approche en composition ait débuté bien avant 2004, au moment où l'orchestre symphonique disparaît. Il arrivait parfois à Baillargeon d'utiliser des cartes Sample Cell, c'est-à-dire des cartes de mémoire dédiées au logiciel Digidesign qui permettaient d'assigner des canaux MIDI à des banques d'échantillons numériques, afin d'épaissir la section de cordes, déséquilibrée par rapport aux cuivres, et de jouer les parties de synthétiseur lorsque la partition l'exigeait ${ }^{36}$. Le compositeur doublait en fait certaines parties des instruments de cette section. Mais en utilisant une machine pour écrire la partition de cordes réelles, limitée par rapport aux instruments acoustiques, Baillargeon a peut-être mis en marche une subtile mutation dans son écriture, comme le croit Simon Leclerc.

Leclerc s'est souvenu d'une discussion qu'il a eue avec Baillargeon au sujet de l'écriture musicale. Le compositeur s'interrogeait sur sa façon d'écrire, sur son approche lorsqu'il devait écrire une partition pour Star Trek. Même si Baillargeon écrivait ultimement pour l'orchestre, Leclerc voyait un danger dans le fait que le compositeur utilise Sample Cell. Progressivement, l'écriture risquait de se modifier, de s'adapter en fonction du timbre des échantillons et aussi des limites de leurs articulations. C'est ce qui s'est partiellement concrétisé avec l'utilisation des échantillons.

Paul Baillargeon reconnaissait qu'avec l'ordinateur et les échantillons, d'autres compositeurs pouvaient être très créatifs, parce que les systèmes permettaient une quantité importante de possibilités ainsi qu'une bonne flexibilité.

34 Paul Baillargeon ne se servait pas de logiciels d'écriture musicale comme Finale ou Sibelius. Toutes les partitions réalisées pour les différents épisodes de Star Trek (sauf les trois derniers) furent écrites à la main.

35 Durant plusieurs années, Paul Baillargeon dirigeait sa propre musique lors des enregistrements de l'orchestre à Los Angeles. Un concours de circonstances amena Simon Leclerc à le remplacer jusqu'à ce que l'orchestre disparaisse.

36 Marc Ouellette (aujourd'hui compositeur, il fut lecteur et assistant technique pour Paul Baillargeon durant ses premières années de travail pour la compagnie Paramount), communication personnelle, 6 octobre 2010. 
Mais l'un des principaux problèmes pour simuler efficacement l'orchestre était le temps requis pour réaliser subtilement les nuances. Le paramétrage était long à effectuer, malgré la courbe d'apprentissage et l'expérience acquise avec le temps. Baillargeon était familier avec l'usage des synthétiseurs, mais il était incapable d'ajuster tous les paramètres du système afin d'obtenir la meilleure performance possible. Il y avait tout simplement trop d'informations à considérer dans le jeu instrumental et les sons réels par rapport aux échantillons de l'orchestre ${ }^{37}$. Le compositeur en était venu à consacrer plus de temps à l'aspect technique de son travail avec les machines, qu'à la composition de sa musique proprement dite.

Il n'a composé pour l'orchestre virtuel que la musique de trois épisodes, soit à peu près $7 \%$ de la production totale de ses musiques pour Star Trek. Mais déjà, sa musique changeait subtilement. Selon l'expérience du compositeur et arrangeur Pierre-Daniel Rheault, les compositeurs habitués à écrire pour l'orchestre traditionnel éviteront d'investir beaucoup de temps à raffiner, perfectionner ou optimiser certaines articulations en utilisant les paramètres offerts sur des logiciels tel que $\mathrm{PLAY}^{3}$. Par ailleurs, les délais de production pour réaliser ces simulations dans le domaine de la télévision sont beaucoup trop courts pour permettre un travail de haute précision. Certains compositeurs auront ainsi tendance à composer de façon moins subtile et, si possible, à réutiliser du matériel existant, aisément disponible une fois créé.

\section{L'EFFET DES TECHNOLOGIES}

L'orchestre symphonique était au cœur de la création musicale des séries Star Trek. Les séances d'enregistrement étaient des évènements collaboratifs importants qui marquaient l'une des dernières étapes créatives de la postproduction d'un épisode. À partir de 2004, Paul Baillargeon commença à utiliser les échantillons numériques pour produire sa musique et il en composa pour trois épisodes de la quatrième et dernière saison de la série Enterprise (2001). La disparition de l'orchestre n'était pas souhaitée et les producteurs auraient préféré maintenir cette tradition musicale au sein des séries télévisées. L'effectif des musiciens a fondu sous les contraintes budgétaires, laissant tout de même le choix aux compositeurs de réaliser leur musique de façon hybride. La qualité des sons numérisés était désormais satisfaisante pour les producteurs et Paul Baillargeon a choisi de ne travailler qu'avec les échantillons.

Cette contrainte avait pour objectifs de réduire les coûts d'opérations pour la production d'Enterprise, mais il fallait s'assurer de la qualité du produit tout en restant aussi fidèle que possible au son original de l'orchestre habituel. Selon Peter Lauritson, les coûts concernant la production de la musique elle-même furent réduits de $35 \% 39$.

Les rôles de Baillargeon se multiplièrent. Bien qu'il ne dirigeait plus l'orchestre depuis des années, remplacé par son collègue Simon Leclerc, il s'est

37 Paul Baillargeon, conférence donnée à l'Université de Montréal, le 2 novembre 2008.

38 Pierre-Daniel Rheault, communication personnelle, 11 août 2009.

39 Peter Lauritson, communication personnelle, 5 décembre 2010. 
remis en quelque sorte à cette activité, car c'était évidemment lui qui devait s'assurer que l'orchestre virtuel sonne comme l'orchestre acoustique. Il a délaissé la baguette du chef pour la souris, le clavier, l'écran à cristaux liquides et quelques logiciels. En plus d'être compositeur de musique pour images, Paul Baillargeon était devenu technicien, un champ d'expertise qui n'était pas nouveau pour lui, mais qui exigeait énormément de temps et d'aptitudes.

Le compositeur assumait également le rôle de l'instrumentiste qu'il devait simuler. Ce qui se retrouvait dans la version définitive d'un top, c'était son propre jeu, ou sa propre programmation ${ }^{40}$. Comme il avait la responsabilité complète du produit final, il était à la fois réalisateur et producteur. Ce transfert de rôles découlant des changements technologiques en production musicale a été soulevé par Robert Ellis-Geiger dans sa thèse de doctorat ${ }^{41}$.

Baillargeon consacrait moins de temps pour créer et pour réfléchir à la musique elle-même qu'il n'en prenait pour l'arranger et la rendre crédible afin qu'elle remplisse ses principales fonctions. Il accordait ainsi plus de temps aux machines qu'à la musique. Richard Winquest s'exprime ainsi à propos de ces changements.

Je crois que si vous demandez à Paul si c'est plus facile d'écrire une partition pour orchestre ou de produire sa musique au synthétiseur, [...] il dirait que c'est plus facile pour lui d'écrire une partition pour orchestre, parce qu'il était en accord exclusivement avec la musique au lieu d'avoir à être toujours préoccupé par cette technologie. Il allait [aux séances d’enregistrement] avec la partition, il lisait la musique, il regardait les musiciens, et il pouvait effectuer des modifications sur place, et c'était fait. Littéralement, ce qui pouvait prendre des heures, des jours [à faire] au synthétiseur pouvait être fait en dix minutes, vous le savez, sur le podium. Donc, la différence énorme pour lui, je pense, c'est qu'il devait être un informaticien pour produire sa musique ${ }^{42}$. (Richard Winquest, communication personnelle, 15 janvier 2010)

Malgré tout, Baillargeon s'adaptera rapidement à tous ces changements. Les échantillons de l'orchestre étaient d'excellente qualité et il réussissait à simuler l'orchestre comme demandé. Il apprit à bien connaître les forces et les faiblesses de ses nouveaux instruments. En utilisant les machines, Paul Baillargeon était parfaitement conscient qu'il écrivait pour « un autre genre d'orchestre ${ }^{43}$ »,

40 Par programmation, nous entendons ici la sélection des articulations des échantillons numériques, le paramétrage des diverses fonctions disponibles sur ses logiciels, le prémixage, etc.

41 Ellis-Geiger 2007, p. 33-36.

42 «I believe that if you would ask Paul if it's easier to write a score for the orchestra or to do a synth score, $[. .$.$] he would say that it's easier for him to write a score with the orchestra, because he$ was in tune strictly with the music, instead of having to be always concerned about this technology. He went out there with the score, he was looking at the music, he was looking at the musicians, and he could change things on the spot, and it was done. Literally, something that could take hours, days on the synthesizer could be done in ten minutes, you know, out on the podium. So, the huge difference for him, I think, is that he had to be a computer scientist to do a score.»

43 Paul Baillargeon, communication personnelle, 4 décembre 2009. 
comme l'ont aussi reconnu des compositeurs tels que James Newton Howard44, réputé pour la qualité exceptionnelle de ses maquettes, et Christophe Beck ${ }^{45}$.

Même si l'on a enlevé aux compositeurs leur principal instrument de musique, l'orchestre symphonique, la sonorité et le genre musical qu'exigeaient d'eux les producteurs devaient rester sensiblement les mêmes. L'écriture devait refléter les textures usuelles de cette musique, les changements subtils d'ambiances, les longues pédales dans le registre grave, les agrégats, les tensions et les détentes, les courtes jonctions entre deux scènes, etc. Toutefois, quelques changements profonds se produisirent dans la confection de la musique de Paul Baillargeon.

\section{Changements STylistiQues}

Nous n'avons pas eu accès aux fichiers MIDI des tops des trois épisodes mentionnés précédemment. Cela nous aurait permis de les comparer avec ceux des épisodes antérieurs que nous avons consultés sous forme de partitions manuscrites. Il aurait alors été plus aisé de valider ce que nous a dit Baillargeon lors de notre dernière rencontre ${ }^{46}$, c'est-à-dire "dès que l'on essaie d'être musicalement subtil avec les machines, cela devient irréalisable». Ainsi, une partie de cette richesse du jeu instrumental humain disparaît avec les machines. Le compositeur a donc écrit une musique sensiblement différente avec les échantillons que ce qu'il aurait souhaité faire avec de vrais instruments. Cela n'a pas changé le genre de musique en soi, mais a modifié quelques aspects stylistiques. Nous avons demandé à Baillargeon de nous montrer un exemple de ce qu'il n'aurait pas fait avec des machines, à partir d'une partition qu'il a lui-même choisie.

Dans l'exemple présenté à la figure 1, tiré du top M42 de l'épisode The Darkling (Voyager), nous avons transcrit les mesures 49 à 52 de la partie d'altos. Le tempo est fixé à 156 battements par minute. À la mesure 49, le motif répété de quatre doubles-croches liées (appelons-le motif a) se transforme par augmentation en croches liées, aux mesures 50 et 51. Ces croches préparent l'exécution du même motif, un ton plus haut cette fois, que l'on retrouve à la mesure 52 . Ce nouveau motif est articulé de la manière suivante: accent sur la première croche, liée à la seconde croche, mais staccato 47 . Cette articulation accent liée staccato de la cellule sol-fa\# (que nous appellerons, motif $b$, peu importe l'intervalle, et qu'il soit ascendant ou descendant), Baillargeon n'en ferait jamais avec des machines. Cela représente beaucoup trop d'ouvrage, dit-il, et ça ne s'approcherait pas de l'effet rebondissant que produiraient les vrais altos.

\footnotetext{
44 «You shouldn't approach MIDI orchestration with the same instrumentation guidelines you'd use for a real orchestra. That's part of the secret to making mockups sound real. » (Karlin 2004, p. 102)

45 "You can't write for the orchestra and then mock it up. You really have to write for the limitations of the sounds you have.» (Karlin 2004, p. 103)

46 Le 15 novembre, 2010.

47 Notez que sur la partition du compositeur, les croches sont groupées par deux, et non par quatre, comme sur notre transcription.
} 


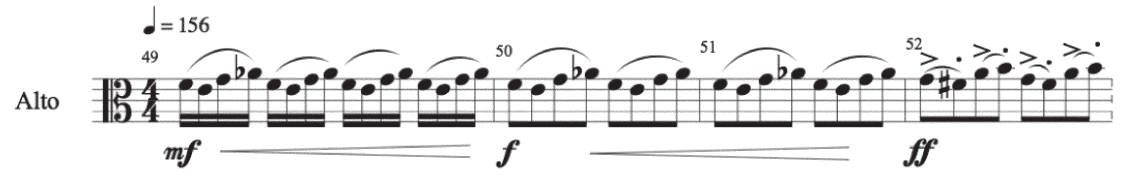

Figure 1. Extrait du top M42 de l'épisode The Darkling. Source : transcription personnelle.

Les motifs $a$ et $b$ reviennent à plusieurs reprises au courant de l'épisode et nous avons constaté que dans le top M44, par exemple, le tempo atteignait 168 $\mathrm{BPM}$. Ces motifs se chantent très bien et un altiste les joue sans problème, comme le disait Paul Baillargeon, et comme en fait foi l'enregistrement de l'orchestre que nous avons écouté. Avec les machines, "ça sonnerait trop mou", même si c'était la clarinette qui les jouerait ${ }^{48}$. En effet, dans la partition originale, les clarinettes doivent jouer ce motif, mais il n'a pu trouver dans ses banques de sons les articulations appropriées pour reproduire cela de façon satisfaisante.

Même s'il était théoriquement possible de reproduire cette combinaison d'articulations en les décomposant et en ajustant avec soin chaque échantillon à l'aide des paramètres du logiciel afin que le tout donne l'impression d'un seul geste musical, le temps à y consacrer amènerait Baillargeon à écrire tout simplement autre chose.

Quand il écrivait sa musique pour orchestre, Paul Baillargeon n'avait qu'à penser à ce qu'il voulait obtenir comme mélodie, comme accords, comme sonorité, etc., et il rédigeait le tout sur papier à musique. Il doit maintenant penser à l'exécution de cette mélodie, à faire varier la sonorité des timbres selon la qualité des banques de son, à ajuster les dynamiques qui peuvent être différentes entre les échantillons d'un même instrument, etc. Et pour y parvenir, selon les modes de jeu choisis, il doit construire une phrase musicale à partir d'éléments discontinus (l'ensemble des échantillons), contrairement à l'interprète qui jouait d'un trait cette phrase, au fur et à mesure que se déroulait la pièce. Cela prend considérablement de temps. Et ce temps qu'il faut consacrer à construire minutieusement une ligne mélodique ou différents modes de jeu était peut-être proportionnel à la connaissance des possibilités offertes par les échantillons, et aussi à l'habileté de l'usager à utiliser les outils informatiques. Il fallait que le temps investi soit rentable par rapport au résultat, étant donné le contexte de son travail et les délais imposés. C'est l'un des premiers problèmes que tous les compositeurs de Star Trek habitués à composer sur papier à musique ont rencontrés lorsqu'ils ont commencé à utiliser les machines. S'il faut «trop» de temps pour produire un passage intéressant, mais complexe, il n'est pas étonnant de constater qu'à la longue, l'écriture se simplifiera.

Dans ce même extrait, à la figure 1, Baillargeon n'utiliserait pas non plus de doubles-croches de cordes à cette vitesse, car l'orchestre virtuel sonnerait trop embrouillé, selon lui. Ailleurs, dans d'autres tops de la même partition, des passages semblables sont joués encore plus rapidement. De plus, dans le cas que nous a décrit le compositeur, trois changements de nuances se produisent

48 Communication personnelle, 15 octobre 2010. 
durant l'extrait. Reproduire ce crescendo, comme le feraient les musiciens de l'orchestre, est techniquement possible, mais musicalement peu souhaitable si l'on tente de simuler un véritable orchestre. Pour passer d'une nuance donnée à une autre, le système utilisé par Baillargeon faisait jouer de nouveaux échantillons, dont la puissance de jeu était plus intense, comme le ferait un instrumentiste. Sauf que, dans le cas de l'instrumentiste, c'est tout le son de l'instrument qui se modifie avec le changement dynamique. Entre les deux nuances standardisées du système virtuel toutefois, c'était le volume d'un échantillon qui augmentait, pas l'intensité du jeu. Bref, c'est le même son qui joue plus fort. Cela passe toujours, étant donné le contexte dans lequel sera jouée la musique (avec piste d'effets sonores, dialogues, etc.), mais, encore une fois, le facteur temps entre en jeu si l'on veut adéquatement simuler l'orchestre réel.

Rapidement, Paul Baillargeon va privilégier une écriture avec des valeurs relativement plus longues, comme la noire. Mais nous tenons à rappeler que le tempo de cet exemple se situe à près de 160 BPM. Nous croyons que ce que nous a raconté Baillargeon s'appliquerait surtout aux mouvements rapides et non aux mouvements lents ou modérés, car les exemples ont préalablement été choisis parmi les tops où il y avait beaucoup d'action, c'est-à-dire des tops où la musique était instrumentalement chargée et très animée. Il semble que les problèmes mentionnés plus haut ne s'appliquaient donc pas aux tops plus lents où le jeu legato, les longues pédales et les articulations les plus simples à reproduire étaient favorisés.

Voici un autre exemple que Paul Baillargeon ne ferait pas à l'ordinateur. Dans ce même épisode, The Darkling, durant le top M53, de la mesure 11 à 15 et dans la portée des violons I, une note manuscrite indique fast gliss at random. Les violons I et II devaient jouer l'agrégat de départ $m i$, fa, sol, la et $s i^{4}$, conserver le $s i$ durant les cinq mesures et effectuer aléatoirement des glissandi à partir des autres notes. Ce genre d'effet, très saisissant et aux sonorités mystérieuses lorsqu'il est joué par un véritable orchestre, perd son efficacité lorsqu'il est construit artificiellement. Les glissandi programmés avec des échantillons numériques seront toujours joués parfaitement, mais sonneront de façon mécanique, à moins de travailler minutieusement un tel passage.

Ces quelques exemples illustrent simplement le fait que le compositeur travaillait avec les instruments virtuels qu'il avait à sa disposition, et que ceux-ci étaient limités et ne pouvaient pas toujours exprimer les idées musicales avec lesquelles il écrivait sa musique auparavant. L'impact des changements technologiques sur la musique de Paul Baillargeon, dans le contexte de Star Trek, fut donc relativement subtil. Mais ces quelques exemples démontrent comment Baillargeon a adapté l'écriture de sa musique orchestrale en fonction de ses nouveaux outils.

Même en tenant compte de la courbe d'apprentissage, qui augmente avec l'usage, et malgré que Paul Baillargeon soit familier avec l'usage des synthétiseurs, il a reconnu qu'il ne pouvait obtenir la meilleure performance possible avec ses appareils parce qu'il y avait trop d'informations à gérer, trop d'éléments à paramétrer. Une partie des difficultés à reproduire ou à simuler l'orchestre provient nécessairement de l'usager. 


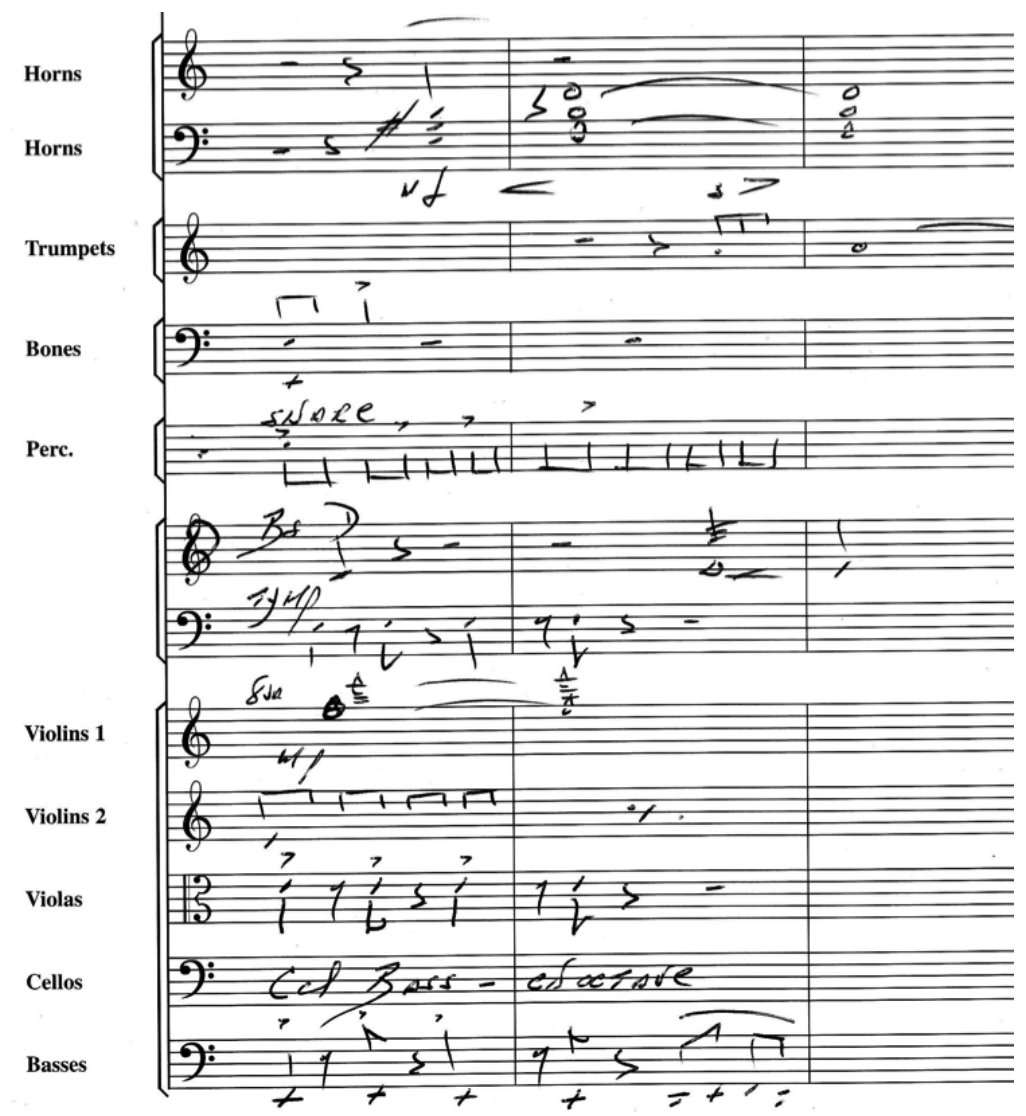

Paul Baillargeon SOCAN (Canada)

Figure 2. Exemple d'une esquisse récente de Paul Baillargeon. Source: Paul Baillargeon, 2010.

À cause des limitations techniques de ses nouveaux outils et du temps nécessaire requis pour les manipuler de façon optimale, Baillargeon a écrit différemment sa musique. Comme il l'a mentionné lors de notre dernier entretien, Paul Baillargeon n'a rien changé à la structure harmonique de sa musique ni au genre qu'il avait pratiqué tout au long de sa carrière chez Paramount, mais il en a simplifié la dimension rythmique, particulièrement pour les tops qui avaient pour fonction de suivre les mouvements de l'action.

Baillargeon fut catégorique durant notre dernier entretien, l'exemple qu'il nous a montré plus haut n’aurait jamais été composé pour un épisode comme Demons (Enterprise), le dernier épisode pour lequel il écrira de la musique. "J'aurais fait une musique plus staccato [il chante la formule rythmique brève brève longue], et j'aurais doublé le tout avec des percussions et aussi de la batterie pour donner de l'impact à l'ensemble 49 ». En effet, tout au long de l'épisode Demons, il s'est abondamment servi de l'anapeste comme motif rythmique.

49 Paul Baillargeon, communication personnelle, 15 novembre 2010. 
À la figure 2, nous avons partiellement reproduit une partition écrite rapidement en guise d'exemple par Paul Baillargeon, avant la fin de notre entretien. Il voulait illustrer ce style de musique, surtout fondé sur des motifs rythmiques simples, qu'il ferait à nouveau pour ce type d'épisodes, particulièrement dans les scènes d'action ou de suspense. Ici, il a écrit un motif rythmique syncopé joué sur mi par les cordes graves (altos, violoncelles et contrebasses), des notes qui sonnent très bien à l'aide de ses échantillons, dit-il, sans doute parce qu'elles sont jouées par des cordes ouvertes. Le mi des violons II produit un fond rythmique stable et la pédale de septième majeur $f a-m i$ dans le registre aigu des violons I crée cette tension caractéristique que l'on retrouve souvent dans sa musique pour Star Trek. Ce n'est qu'un court exemple, griffonné à la hâte, mais qui donne le ton à ce nouveau style simplifié adopté par Baillargeon à la fin de son travail pour Paramount.

Par ailleurs, l'un des effets de l'écriture de tops à l'aide du séquenceur des logiciels de production musicale fut la réutilisation du matériel déjà produit, ce qui ne se faisant auparavant que dans de très rares cas, et seulement par l'éditeur musical. En télévision, il est maintenant courant et naturel de reprendre du matériel existant, de le transformer et de le reproduire différemment en modifiant l'une ou plusieurs de ses caractéristiques. C'est le principe même de la variation et cela s'applique très bien à l'aide des technologies numériques. En effet, c'est ce que Baillargeon a fait pour quelques passages du dernier épisode. Il s'est servi de sa propre musique, créée pour l'un des deux premiers épisodes où la musique fut produite à l'aide des machines. Avec l'ordinateur et les outils de production actuels, il est aisé d'adapter un top existant à une nouvelle scène, d'en modifier l'instrumentation, le tempo et une multitude d'autres paramètres.

Paul Baillargeon n'a pas eu l'opportunité de pousser plus à fond son expérience avec les machines dans le cadre de Star Trek puisque la série Enterprise s'est terminée l'année même où les compositeurs durent s'adapter aux nouvelles réalités économiques de la série. Même s'il n’a composé de cette façon que pour trois épisodes, nous avons constaté de subtils changements dans le style de son écriture et, compte tenu des tendances que nous avons remarquées, il n'est pas impossible de croire qu'à la longue, sa musique se serait de plus en plus uniformisée, laissant de moins en moins de place à sa créativité.

\section{Conclusion}

Les pressions économiques liées à la production télévisuelle et à la qualité croissante des technologies numériques ont affecté les différents processus de postproduction des séries Star Trek, comme par exemple celui de la création de la musique. L'orchestre symphonique, cet ensemble unique et vivant qui impliquait un niveau de collaboration élevé entre de nombreux intervenants, a disparu au profit des systèmes de création musicale assistés par ordinateur fondé sur l'échantillonnage. Certains rôles dédiés à différents acteurs se sont ajoutés au travail de Paul Baillargeon, comme celui d'interprète et de technicien. Les machines imposent à l'usager l'acquisition des savoirs nécessaires 
à leur manipulation. Il n'y a plus de transparence entre le compositeur et la musique que celui-ci conçoit, mais une interface standardisée, aux applications uniformisées. Malgré cette forme de limitation, les outils numériques offrent de nombreux avantages dans un contexte où la musique sert d'arrière-plan à la trame de l'histoire présentée à l'image, dans un contexte où le temps et l'argent doivent être contrôlés le plus efficacement possible.

Ces impacts technologiques sur la musique sont, paradoxalement, "naturels»; le musicien compose pour les instruments qu'il a à sa disposition. Par ailleurs, au-delà des contraintes imposées par les machines et les méthodes requises pour les utiliser, l'usage des technologies numériques touche nécessairement à la musique elle-même. Il est difficile d'en mesurer la grandeur de façon précise dans le cas de Baillargeon, mais les témoignages recueillis confirment leur existence. Certains de ces changements sont profonds et difficiles à cerner, relevant du processus de composition lui-même, affectant avec nuance son style musical. Mais ce que représente aussi l'histoire de Paul Baillargeon dans le contexte de Star Trek, c'est la fin d'une époque qui perpétuait un savoirfaire en vigueur depuis plus de soixante-dix ans. Le développement continuel des technologies musicales rend impossible un retour à l'usage de l'orchestre symphonique en télévision et annonce peut-être la disparition éventuelle des grands ensembles acoustiques dédiés à la musique pour image en mouvement.

\section{RÉFÉRENCES}

Baillargeon, Paul. 4 décembre 2009. Entretien personnel entre Paul Baillargeon et François Gauthier. Lachute (Québec).

—. 17 septembre 2009. Entretien personnel entre Paul Baillargeon et François Gauthier. Lachute (Québec).

—_. 27 août 2009. Entretien personnel entre Paul Baillargeon François Gauthier. Lachute (Québec).

—. 15 novembre 2010. Entretien personnel entre Paul Baillargeon et François Gauthier. Lachute (Québec).

_.15 octobre 2010. Entretien personnel entre Paul Baillargeon et François Gauthier. Lachute (Québec).

Berman, Richard. 24 novembre 2010. Entretien téléphonique entre Richard Berman François Gauthier. Montréal/Los Angeles.

Bond, Jeff. 1998. The Music of Star Trek: Profiles in Style. Los Angeles, CA: Lone Eagle Publishing Company.

Ellis-Geiger, Robert Jay. 2007. "Trends in Contemporary Hollywood Film Scoring: A Synthesized Approach for Hong Kong Cinema.» Thèse de doctorat, Leeds, The University of Leeds, School of Music.

Heinrich, Marie-Noëlle. 2003. Création musicale et technologies nouvelles : mutation des instruments et des relations. Paris : L'Harmattan.

Karlin, Fred \& Wright, Rayburn. 2004. On the Track: A Guide to Contemporary Film Scoring. New York, NY : Routledge.

Lauritson, Peter. 5 décembre 2010. Entretien téléphonique entre Peter Lauritson et François Gauthier. Montréal/Los Angeles. 
Leclerc, Simon. 19 novembre 2009. Entretien personnel entre Simon Leclerc et François Gauthier. Ste-Agathe (Québec).

Ouellette, Marc. 6 octobre 2010. Entretien personnel entre Marc Ouellette et François Gauthier. Longueuil (Québec).

Ottens, N. 2007. Site web Forgotten Trek. http://www.ottens.co.uk/forgottentrek /tng_8.php. [consulté le 31 janvier 2012].

Rheault, Pierre-Daniel. 11 août 2009. Entretien personnel entre Pierre-Daniel Rheault et François Gauthier. Montréal (Québec).

Winquest, Richard (15 janvier 2010). Entretien téléphonique entre Richard Winquest et François Gauthier. Montréal/Los Angeles.

\begin{abstract}
Paul Baillargeon, a Canadian, composed the music for more than forty Star Trek television episodes, which are now translated and known around the world. Star Trek is one of the last American television series to have its music played by an important symphonic orchestra, before composers understood that they could use instead computers and digital samples, replacing by doing so the fifty musicians who were perpetuating the acoustic musical Hollywood tradition. This essay examines how those digital tools had impacted on composers' music, and on his work within the postproduction stages of popular television series.
\end{abstract}

\title{
RÉSUMÉ
}

Le compositeur canadien Paul Baillargeon a réalisé la musique de plus de quarante épisodes des séries télévisées Star Trek, maintenant traduites et diffusées à travers le monde. Star Trek est l'une des dernières séries télévisuelles américaines ayant utilisé un orchestre symphonique d'envergure avant que ses principaux compositeurs réalisent eux-mêmes leurs bandes sonores à l'aide d'ordinateurs et d'échantillons numériques, remplaçant ainsi la cinquantaine de musiciens qui perpétuait la tradition acoustique de la production musicale à Hollywood. Cet article explore comment ces outils ont influencé la musique du compositeur, ainsi que son travail au sein de la postproduction des populaires séries télévisées. 